\title{
Desequilíbrios musculares entre flexores dorsais e plantares do tornozelo após tratamento conservador e acelerado da ruptura do tendão calcâneo Muscle imbalance between ankle dorsiflexors and plantarflexors after conservative and accelerated treatment of Achilles tendon rupture
}

\author{
Alexandre Mayer ${ }^{1}$, Viviane Bortoluzzi Frassonn', Rafael Ott ${ }^{3}$, Rafael de Oliveira Fortuna ${ }^{4}$, Marco Aurélio Vaz
}

Estudo desenvolvido no Setor de Plasticidade Neuromuscular do Laboratório de Pesquisa do Exercício da Esef/UFRGS Escola de Educação Física da Universidade Federal do Rio Grande do Sul, Porto Alegre, RS, Brasil

1 Fisioterapeuta; Prof. Especialista da Esef/UFRGS

2 Fisioterapeuta; Profa. Ms. da PUCRS - Pontifícia Universidade Católica do Rio Grande do Sul

3 Médico Ms. do Serviço de Ortopedia e Traumatólogia do Hospital São Lucas da PUCRS

4 Fisioterapeuta; doutorando na University of Calgary, Calgary, AB, Canadá

5 Educador físico; Prof. Dr. da Esef/UFRGS

ENDEREÇO PARA CORRESPONDÊNCIA

Alexandre Mayer Laboratório de Pesquisa do Exercício/ Esef/UFRGS R. Felizardo 750 Jardim Botânico

90690-200 Porto Alegre RS e-mail:

mayer_puc@yahoo.com.br

Este estudo recebeu apoio financeiro do Curso de Especialização em Cinesiologia da Esef/UFRGS

ApRESENTAÇÃo maio 2009

ACEITO PARA PUBLICAÇÃO abr. 2010
Resumo: A ruptura do tendão calcâneo (TC) reduz a sobrecarga mecânica dos flexores plantares (FP) do tornozelo. Essa alteração muda o equilíbrio natural entre os FP e flexores dorsais (FD) do tornozelo. O objetivo do estudo foi avaliar as razões isocinéticas concêntricas convencionais de torque de pacientes submetidos a tratamento cirúrgico de ruptura aguda do TC após dois protocolos diferentes de reabilitação. Após procedimento cirúrgico para reconstrução do TC, a amostra foi dividida de forma intencional em dois grupos: conservador (GC, 11 homens, $41,3 \pm 7,9$ anos) e grupo acelerado (GA, 13 homens, 43,5 $\pm 13,7$ anos). O GC permaneceu com imobilização gessada no tornozelo por seis semanas (tratamento tradicional), enquanto o GA usou uma órtese robofoot em posição neutra e, após duas semanas, iniciou mobilização e apoio precoce do tornozelo, com reabilitação por seis semanas. Após 3 meses de pós-operatório, a razão do torque concêntrico máximo dos FD pelos FP do tornozelo foi avaliada por dinamômetro isocinético. As razões de torque do lado operado se mantiveram superiores às do lado saudável mesmo após 3 meses de pós-operatório $(p<0,05)$. Não foi encontrada diferença no equilíbrio muscular entre os grupos. Pode-se concluir que os dois grupos requerem um período mais longo de reabilitação para recuperar o equilíbrio natural do tornozelo no lado operado.

Descritores: Músculo esquelético; Ruptura/reabilitação; Tendão do calcâneo/ lesões; Torque

ABSTRACT: Achilles tendon rupture reduces ankle plantarflexor (PF) muscles mechanical overload. This change in the ankle joint mechanics changes the natural muscle balance between dorsiflexor (DF) and PF muscles. The purpose of this study was to assess such imbalance by concentric conventional isokinetic torque ratios of patients who underwent different rehabilitation protocols after surgical repair of the Achilles tendon. After surgery, subjects were assigned to either a conservative or to an accelerated rehabilitation group. The conservative group (11 men, $41.3 \pm 7.9$ years old) remained with a plaster cast for 6 weeks after surgery. The accelerated group (13 men, 43.5 $\pm 13,7$ years old) used a "robofoot" cast for 2 weeks and underwent ankle mobilization and early weight bearing for a period of 6 weeks postimmobilization. At 3 months post-surgery the ratio between the maximal concentric DF torque and the maximal concentric PF torque was evaluated with an isokinetic dynamometer. Torque ratios on the surgery side were higher $(p<0.05)$ than on the sound side in both groups. No differences of muscle balance could be found between the groups. These results suggest that both groups require further rehabilitation to regain the natural muscle balance between surgery-side ankle DF and PF muscles. Key words: Achilles tendon/injuries; Muscle, skeletal; Rupture/rehabilitation; Torque 


\section{INTRODUÇÃO}

O tendão do calcâneo (TC) é o maior e mais resistente tendão do corpo humano. É formado pela porção distal dos músculos gastrocnêmios e solear, e tem a função de transmitir força desses músculos à porção posterior do calcâneo, determinando a flexão plantar (FP) do tornozelo ${ }^{1}$.

As rupturas agudas do TC são relativamente comuns, com uma incidência estimada em 18/100.000 pessoas ${ }^{1}$. Essa incidência aumentou nos últimos 50 $\operatorname{anos}^{1-3}$, devido ao interesse crescente por atividades recreacionais relacionadas ao esporte. Entre 81 e $89 \%$ das rupturas do TC ocorrem durante a prática desportiva $^{1,3}$. Constituem uma fonte substancial de prejuízo social e funcional, afetando primariamente indivíduos adultos do sexo masculino entre a $4^{\underline{a}}$ e $5^{\underline{a}}$ décadas de vida ${ }^{4}$.

Imediatamente após a lesão, acompanhada por estalido, é relatada dor súbita e intensa na porção posterior do tornozelo. Essa dor pode diminuir rapidamente, induzindo o paciente a uma sensação de lesão menor ${ }^{4}$. O diagnóstico ao exame físico pode ser estabelecido pela presença de três achados comuns: diminuição da força de FP do tornozelo, depressão palpável e geralmente visível noTC, e teste de Thompson positivo (que consiste na aplicação de pressão circunferencial à porção mais volumosa dos músculos gastrocnêmio e sóleo ${ }^{4}$ ).

Apesar de atribuir-se a ruptura do TC à atividade esportiva, lesões intrínsecas determinam redução de sua resistência mecânica ${ }^{5}$, contribuindo para a ruptura; em $25 \%$ dos pacientes com rupturas agudas observou-se alguma forma de processo inflamatório prévio em seus tendões ${ }^{5}$. Estudo histopatológico em TCs com ruptura espontânea revelou evidências de lesão prévia (97\% dos casos envolveram degeneração mucóide, lipomatosa, isquêmica ou calcificações, e nos 3\% restantes foi verificada a presença de infiltração tumoral ou artropatia inflamatória), incluindo o uso de esteróides como fator associado às rupturas espontâneas ${ }^{6}$.

O princípio básico do tratamento cirúrgico é a restauração durável da con- tinuidade do TC rompido, proporcionando cicatrização em posição fisiológica e restauração da função muscular ${ }^{7}$. No pós-operatório (PO) clássico ou conservador, a articulação e a tenorrafia (sutura do TC) são protegidos e imobilizados com aparelho gessado por 6 semanas, após as quais se inicia programa de exercícios. Apesar dos benefícios desse método - principalmente em pacientes sem condições de suporte domiciliar para os cuidados $\mathrm{PO}$, pois o gesso assegura a estabilidade do tecido suturado, protegendo-o de eventuais traumatismos ou quedas - a imobilização de qualquer segmento leva a perdas musculares e dos tecidos moles em torno da articulação ${ }^{7}$. Os tecidos conjuntivos fibrosos densos (como ligamentos, tendões) e músculos são sensíveis tanto ao treinamento quando à redução do uso (imobilização) ${ }^{8}$. A supressão e/ou a redução das cargas devido à imobilização produz uma deterioração rápida nas propriedades bioquímicas e mecânicas dos tendões ${ }^{8}$. Além disso, o desuso prolongado gera atrofia e rigidez ${ }^{9,10}$. Em função desses efeitos deletérios da imobilização póscirúrgica, autores vêm preconizando a utilização da mobilização e apoio precoce na reabilitação ${ }^{11-13}$.

Um programa de reabilitação precoce parece devolver aos tecidos musculoesqueléticos as propriedades mecânicas em uma rápida adaptação desses tecidos ao retorno da sobrecarga mecânica. Como os FP e flexores dorsais (FD) do tornozelo recebem demandas ou sobrecargas mecânicas diferentes nas atividades de vida diárias, eles apresentam um desequilíbrio que é natural, decorrente das adaptações às suas demandas funcionais respectivas. Nesse sentido, os FP apresentam maior área de seção transversa fisiológica que os FD, e, portanto, maior capacidade de gerar força.

Uma das formas de avaliar esse desequilíbrio natural é através de razões entre o torque isocinético máximo concêntrico de grupos musculares antagonistas. Esse desequilíbrio fisiológico na atividade muscular é extremamente importante no trabalho de ligamentos, que promovem estabilidade articular e controle do movimento ${ }^{14}$. Em indivíduos sedentários essa razão do torque concêntrico dos FD pelo torque dos FP (obtida em uma ve- locidade angular de $60 \%$ s) é de 0,26 para ambos os lados, dominante (D) e nãodominante (ND) ${ }^{15}$. Entretanto, esse desequilíbrio natural é alterado em atletas de algumas modalidades esportivas em razão das diferentes demandas funcionais a que os mesmos são submetidos durante a prática esportiva ${ }^{14}$. Em ciclistas, por exemplo, a razão de torque concêntrica foi de 0,26 no lado $D$ e de 0,23 no lado ND (semelhante aos valores normais), enquanto em ginastas essas razões são de 0,35 para o lado D e 0,36 para o lado ND (valores superiores aos normais). Jogadores de futebol apresentam valores semelhantes aos ginastas $(0,31$ para o lado D e 0,33 para o lado ND) ${ }^{16}$. Esses resultados demonstram a existência de um aumento do desequilíbrio muscular natural decorrente das demandas funcionais da prática esportiva. Essas razões também são alteradas após intervenções clínicas. Duas semanas de imobilização em sujeitos com fratura de tornozelo, por exemplo, determinaram uma atrofia dos FD em $18,9 \%$ e $24,4 \%$ dos $\mathrm{FP}^{7}$, demonstrando que o processo de redução do uso altera a capacidade de produção de força, que essa alteração ocorre de maneira diferente entre grupos musculares antagonistas, e que essa diferença na redução da força altera as razões de torque do tornozelo. Nesse sentido, a ruptura do TC deveria aumentar as razões convencionais de torque em função da perda de massa muscular (sarcopenia) decorrente do processo de lesão dos FP, que acarreta uma redução do uso desse grupo muscular.

Não foram encontrados na literatura estudos que avaliaram os desequilíbrios musculares dos músculos do tornozelo após sutura do TC ou estudos que avaliaram as razões de torque entre pacientes submetidos a tratamento conservador e pacientes submetidos a um protocolo acelerado de reabilitação dessa lesão. $\mathrm{O}$ objetivo do presente estudo foi avaliar as alterações no desequilíbrio natural da musculatura do tornozelo por meio das razões isocinéticas concêntricas convencionais de pacientes submetidos à cirurgia de reconstrução do TC. Além disso, procurouse determinar se um protocolo acelerado de reabilitação produziria um retorno mais rápido dessas razões a valores normais, quando comparado a um protocolo conservador (imobilização). A 
hipótese inicial do estudo era de que o lado operado apresentaria razões de torque maiores que 0,26 . Além disso, esperava-se que as razões de torque do grupo conservador fossem maiores do que as razões do grupo submetido ao protocolo de reabilitação acelerado, que seriam mais próximas de 0,26 , demonstrando assim um desequilíbrio muscular mais próximo dos valores normais após a reabilitação.

\section{METODOLOGIA}

Todos os procedimentos do estudo foram aprovados pelo Comitê de Ética em Pesquisa do Hospital São Lucas da PUCRS - Pontifícia Universidade Católica do Rio Grande do Sul. Todos os sujeitos assinaram um termo de consentimento esclarecido após receberem as informações referentes aos objetivos do estudo, riscos e benefícios.

Para participar do estudo os sujeitos deveriam ter diagnóstico de ruptura aguda total do TC e sido submetidos a tratamento cirúrgico com método de sutura término-terminal (com quatro pontos loops, como descrito pela técnica de sutura direta Krackow ${ }^{17}$ ), feito pelo Setor de Ortopedia e Traumatologia do Hospital São Lucas da PUCRS. Todos os procedimentos cirúrgicos foram realizados pelo mesmo cirurgião a fim de garantir que a mesma técnica fosse utilizada em todos os pacientes.

A amostra foi dividida de forma intencional em dois grupos: grupo conservador (CON) e acelerado (ACE), segundo a freqüência ou não ao programa de reabilitação: pacientes residentes em outro município foram incluídos no grupo CON, devido à impossibilidade de comparecimento ao hospital para a reabilitação, tendo recebido orientações de exercícios a serem feitos em domicílio; os pacientes do grupo ACE eram residentes na região metropolitana de Porto Alegre que concordaram em participar do programa de reabilitação. O grupo CON foi composto por 11 homens, com

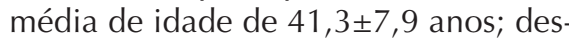
tes, 7 sujeitos apresentaram lesão do lado esquerdo e 4 do lado direito. O grupo ACE foi composto por 13 homens, com idade média de 43,5 513,7 anos, 7 com lesão no lado direito e 6 no lado esquerdo.
Foram excluídos do estudo pacientes com doenças neuromusculares, diabéticos, pacientes que apresentassem qualquer contra-indicação para a realização de teste de força máxima em dinamômetro isocinético e que apresentassem qualquer problema no local de realização da cirurgia, ou pacientes do grupo ACE com mais de duas faltas durante o período de tratamento. Ao final, foram excluídos do estudo três indivíduos do grupo CON: um por apresentar processo infeccioso e dois por não comparecerem à avaliação isocinética aos três meses de pós-operatório.

\section{Procedimentos pós-operatórios}

Após o procedimento cirúrgico, os sujeitos do grupo CON permaneceram com imobilização gessada na articulação do tornozelo durante seis semanas (tratamento tradicional). Após a retirada do gesso, receberam uma ficha demonstrativa dos exercícios a serem feitos em casa e treinamento nesses exercícios. Além disso, foi enfatizada a importância de aderirem ao programa de reabilitação para o restabelecimento mais rápido. Qualquer dúvida ou dificuldade na execução dos movimentos poderia ser sanada por contato telefônico com o fisioterapeuta da equipe de reabilitação. Quando os sujeitos compareceram ao laboratório para a avaliação de três meses de PO, foram questionados sobre a realização dos exercícios conforme orientados. Não foram tomadas medidas adicionais de controle da realização dos exercícios pelos pacientes desse grupo.

Nas três primeiras semanas de reabilitação, o exercícios preconizados para os pacientes do grupo CON foram: ativos livres de FD e FP do tornozelo (3x20 repetições, na posição sentado com o joelho estendido); exercícios de apoio na ponta do pé (3x20), com descarga de peso bipodal, progredindo para unipodal; exercícios de agachamento $(3 \times 10$, evoluindo para $3 \times 30$ ) foram realizados inicialmente com apoio bipodal, passando para apoio unipodal após três semanas de reabilitação (3x20). Exercícios de alongamento para a região posterior da perna do lado operado foram realizados na posição em pé com o membro operado estendido e o tornozelo em FD, enquanto o lado saudável era flexionado e os membros superiores eram apoiados na parede $(5 \times 20)$. A orientação era de fazerem os exercícios todos os dias, por seis semanas, até completarem os três meses de PO.

Os pacientes do grupo ACE usaram uma órtese comercial removível tipo robofoot (Robofoot Nova Geração, Salvapé, SP, Brasil) posicionada no tornozelo em posição neutra. Após duas semanas de PO, os sujeitos do grupo ACE foram submetidos a seis semanas de reabilitação. As atividades do protocolo de reabilitação consistiram em exercícios de flexibilidade (que envolveram a mobilização do tornozelo para ganho de amplitude de movimento), exercícios de resistência muscular localizada com o uso de bandas elásticas thera-band (Thera-Band Europe $\mathrm{GmbH}$, Alemanha) para aumento da resistência, e aumento da sobrecarga pela mudança da thera-band para ganho de força. Todos os pacientes foram submetidos à mesma progressão: a partir da 4a semana de reabilitação ( $6^{a}$ semana de PO), foi acrescentado exercício resistido $(2 \times 15)$ com thera-band vermelha para os movimentos de eversão, inversão, FP, FD, com uma sobrecarga correspondente a 5\% do peso corporal; na $5^{\mathrm{a}}$ e $6^{\mathrm{a}}$ semanas foram mantidas as séries, repetições e movimentos, enquanto a carga foi aumentada para $10 \%$ do peso corporal por meio da utilização de uma thera-band preta. A graduação da thera-band foi baseada em tabela retirada do site dos produtores da thera-band (www.theraband.com), sendo que a cada " $\mathrm{x}$ " $\mathrm{cm}$ de estiramento da faixa corresponde uma carga de " $n$ " $k$, que depende da sua cor, espessura e distensão. A carga utilizada de $5 \%$ na 4 a e $10 \%$ na $5^{\underline{a}}$ e $6^{\underline{a}}$ semanas foi definida pelos pesquisadores, pois não foram encontrados dados na literatura relacionados à reabilitação do TC com o uso da thera-band, ou mesmo com percentuais do peso corporal. Os sujeitos do grupo ACE iniciaram ainda apoio precoce do pé após a $2^{a}$ semana de $\mathrm{PO}$ com exercícios de deambulação com a robofoot, progredindo para descarga total de peso sem a bota na $7 \stackrel{\text { a }}{\text { semana de }}$ PO. Todas as atividades de reabilitação foram realizadas três vezes por semana no Setor de Fisioterapia do Hospital São Lucas. Após o término do programa 
supervisionado de fisioterapia, os pacientes do grupo ACE receberam a mesma ficha demonstrativa dos exercícios do grupo CON, que deveriam ser realizados em casa até completarem três meses de PO.

\section{Avaliação do torque isocinético}

Todos os pacientes foram submetidos a uma avaliação do torque isocinético concêntrico máximo três meses após a cirurgia. Um dinamômetro isocinético Biodex System 3 Pro (Biodex Medical System, NY, USA) foi utilizado para obter os valores do torque isocinético concêntrico máximo dos FP e FD do tornozelo. Os pacientes foram posicionados sentados na cadeira do dinamômetro com o joelho estendido e o pé fixo na prancha de fixação por meio de faixas de velcro. O sujeito foi posicionado de modo que o eixo de rotação aparente da articulação do tornozelo estivesse alinhado com o eixo de rotação do dinamômetro.

Todos os pacientes executaram três contrações voluntárias máximas concêntricas de FP e FD do tornozelo em uma velocidade angular de $60 \%$ s. A avaliação do torque foi feita de forma dinâmica concêntrica para evitar uma sobrecarga mecânica excessiva sobre os tecidos conjuntivos, que poderia ocorrer em uma avaliação excêntrica, e porque as

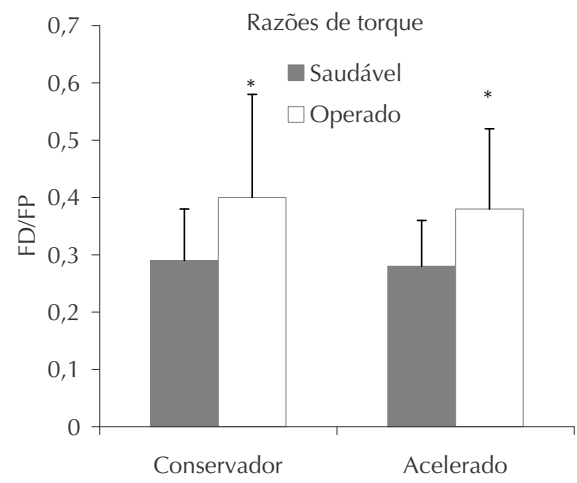

Gráfico 1 Razão de torque (flexores dorsais/flexores plantares, FD/ FP) do tornozelo (média \pm desvio padrão) dos membros saudável e operado dos grupos conservador e acelerado; * $p<0,05$ na comparação entre membro saudável e membro operado contrações concêntricas possibilitam avaliar a capacidade de produção durante uma situação dinâmica e talvez mais fisiológica ou funcional. Um intervalo de dois minutos foi observado entre as contrações máximas a fim de minimizar possíveis efeitos da fadiga. Após a avaliação do membro saudável, o mesmo teste foi repetido no membro operado.

\section{Análise dos dados}

Das três contrações voluntárias máximas concêntricas a contração de maior valor de torque foi utilizada para a análise. Esse valor de torque de cada grupo muscular foi utilizado para a determinação das razões de torque concêntricas convencionais. A análise descritiva dos dados consistiu do cálculo das médias e desvios padrão das médias das razões de torque e do torque máximo dos dois grupos musculares. A normalidade dos dados foi testada com o teste Shapiro-Wilk. Para comparação do torque e das razões de torque entre os lados saudável e operado dentro de cada grupo foi utilizada uma Anova de um fator para dados repetidos. Para comparação das razões de torque de cada lado (saudável e operado) entre os grupos (ACE x CON), foi utilizado o teste $t$ de Student para amostras independentes. $\mathrm{O}$ programa SigmaStat 3.5 foi utilizado

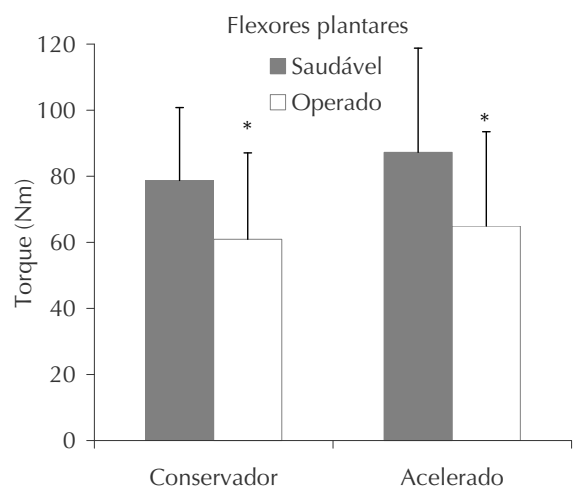

Gráfico 2 Torque isocinético concêntrico máximo (média \pm desvio padrão) dos músculos flexores plantares do tornozelo; * $p<0,05$ na comparação intra-grupo entre membro saudável e operado para todas as análises, com um nível de significância de 5\%.

\section{RESULTADOS}

Foram encontrados maiores valores das razões de torque entre os flexores dorsais e plantares (FD/FP) no membro operado do que no membro saudável (Gráfico 1), tanto no grupo CON $(p=0,023)$ quanto no ACE $(p=0,021)$. As razões de torque se mostraram semelhantes entre os grupos tanto no membro saudável $(p=0,765)$ quanto no membro operado $(p=0,840)$ nas comparações entre os diferentes protocolos (ACE x CON).

Quanto aos flexores plantares, mediramse valores menores do torque isocinético concêntrico (Gráfico 2) no membro operado do que no saudável, tanto no grupo CON $(p=0,004)$ quanto no grupo ACE $(p=0,002)$. Não foi encontrada diferença do torque isocinético concêntrico dos FP entre os lados saudáveis $(p=0,461)$, ou entre os lados operados $(p=0,725)$ dos dois grupos.

Quanto ao torque isocinético concêntrico dos FD, mostrou-se semelhante entre os lados saudável e operado, tanto no grupo $\mathrm{CON}(p=0,354)$ quanto no grupo ACE $(p=0,852)$, e entre os lados saudável $(p=0,588)$ e operado $(p=0,994)$ entre os dois grupos (Gráfico 3). Não foram encontradas diferenças na capacidade de produção de torque entre os lados saudável e operado, nem entre os grupos conservador e acelerado.

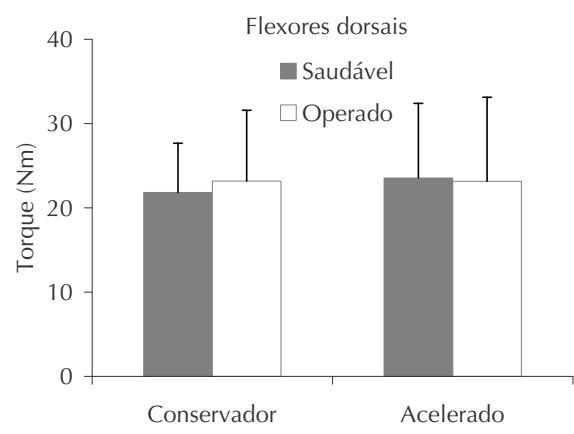

Gráfico 3 Torque isocinético concêntrico máximo (média \pm desvio padrão) dos músculos flexores dorsais do tornozelo 


\section{DISCUSSÃO}

A totalidade dos pacientes do presente estudo foi constituída por sujeitos do sexo masculino entre a $4^{a}$ e $5^{\underline{a}}$ décadas de vida. Essa maior incidência de ruptura do TC nos homens é semelhante à de outros estudos ${ }^{3,5,18}$ e parece estar associada aos chamados "praticantes de esporte de fim de semana". Isso sugere que o mecanismo de lesão tendínea está associado a dois fatores fundamentais: falta de condicionamento físico acarretando fraqueza dos tecidos, e trauma agudo por estiramento dos FP durante contrações excêntricas (onde ocorre a soma das forças externas com as forças musculares intrínsecas) incidindo sobre a junção miotendinosa (local de maior fraqueza do $\mathrm{TC}^{12}$ ).

Os valores das razões de torque do lado saudável foram semelhantes nos dois grupos $(0,29$ no grupo CON e 0,28 no grupo ACE) e semelhantes aos valores de razões de torque de indivíduos sedentários $(0,26)^{15}$. Como o lado saudável não foi submetido a qualquer tipo de intervenção nos dois grupos, essa semelhança era esperada. Entretanto, a utilização do lado contralateral como controle do lado operado deve ser feita com cautela, uma vez que a lesão do TC acarreta um aumento da sobrecarga mecânica sobre o lado contralateral na realização das atividades de vida diária. Além disso, enquanto o grupo CON descarregou todo o peso do corpo no lado saudável assistido por muletas, iniciando o apoio no membro operado somente após seis semanas de PO, o grupo ACE iniciou apoio parcial no membro lesionado com duas semanas de PO, diminuindo dessa forma a descarga de peso no membro saudável quatro semanas antes do grupo CON.

Os maiores valores observados nessa razão do lado operado em relação ao saudável nos grupos $\operatorname{CON}(0,40)$ e ACE $(0,38)$ demonstra um desequilíbrio muscular entre os FD e FP do tornozelo, confirmando a primeira hipótese do estudo, de que deveria ocorrer um aumento da razão no lado operado quando comparado ao contralateral saudável. Esse maior valor das razões de torque ocorreu em função da já esperada redução na capacidade de produção de força dos FP (Gráfico 2), decorrente da imobilização e redução da sobrecarga mecânica às quais os FP ficam sujeitos depois da lesão e cirurgia. Além disso, há uma manutenção do torque dos FD (Gráfico 3). Como esse grupo muscular não sofre lesão e não reduz sua capacidade de produção de força, por ser pouco utilizado no dia-a-dia para sustentar peso, não sofre qualquer influência da imobilização.

Apesar dos benefícios da imobilização, como a proteção e prevenção de rupturas dos tecidos em fases iniciais de cicatrização até a restauração de sua integridade, a principal conseqüência desse procedimento clínico é a redução na capacidade de produção de força muscular decorrente de perda de massa muscular ou sarcopenia ${ }^{19}$. Já se sabe que a imobilização afeta principalmente a musculatura mais utilizada no dia-a-dia para a descarga de peso, como é o caso do músculo gastrocnêmio, responsável por $90 \%$ da força dos FP durante a caminhada ${ }^{14}$.

O fato de as razões de torque se apresentarem elevadas no lado operado dos dois grupos mostra que, mesmo depois de três meses de PO, não houve recuperação da funcionalidade dos FP do tornozelo. Isso também alerta para a temporalidade do processo de recuperação da lesão no TC, que pode levar de 6 meses a 1 ano para o retorno aos níveis funcionais do lado saudável. A conscientização dos pacientes é portanto fundamental de que o processo de reabilitação é lento, contínuo e demorado.

A semelhança nas razões de torque do lado operado entre os grupos (hipótese 2) não era esperada, uma vez que a sobrecarga mecânica se iniciou antes no grupo ACE - que iniciou a descarga de peso no lado operado durante as sessões de reabilitação. Existem duas possibilidades para explicar esses resultados: (1) a sobrecarga mecânica aplicada ao grupo ACE foi insuficiente para produzir uma recuperação precoce nesse grupo; ou (2) a sobrecarga mecânica das atividades de vida diária e dos exercícios domiciliares realizados nas semanas que antecederam o teste de força (que ocorreu com 12 semanas de $\mathrm{PO}$ ) foi suficiente para produzir razões de torque similares entre os grupos.
Segundo a literatura ${ }^{13}$, um deficit de $34 \%$ foi observado na comparação entre membro saudável e lesionado (em uma velocidade angular de $60 \%$ s) após três meses de reabilitação. O deficit de $24 \%$ encontrado nos dois grupos do presente estudo sugere que tanto o programa de exercícios orientados na clínica (grupo ACE) quanto o de exercícios realizados no próprio domicílio (grupo CON) reduziu o deficit bilateral. Diversos estudos ${ }^{12,13,20-23}$ concluem que a mobilização e cargas precoces tornam o tendão mais resistente, devido ao aumento no conteúdo de colágeno e orientação na deposição das fibrilas de colágeno na mesma linha de ação do músculo. Esses fatores reduziriam a possibilidade de ocorrência de novas rupturas, justificando a utilização de reabilitação precoce ou acelerada como segura e benéfica ao paciente, permitindo-lhe uma recuperação mais rápida e com menor incidência de seqüelas.

Este estudo parece ser o primeiro a avaliar os desequilíbrios musculares entre FD e FP do tornozelo de pacientes pós-ruptura do TC. Além disso, a avaliação precoce (três meses de $\mathrm{PO}$ ) do torque foi apenas relatada em um estu$\mathrm{do}^{24}$, pois esse tipo de avaliação poderia acarretar riscos para pacientes submetidos à sutura do TC. No entanto, nenhuma reruptura ou complicação decorrente do teste foi encontrada, indicando que a avaliação precoce do torque é segura para determinar as alterações musculares devidas a essa cirurgia.

A eficácia do programa acelerado de fisioterapia não foi constatada neste estudo, sugerindo que a sobrecarga utilizada não foi suficiente para produzir a recuperação da produção de força dos FP do tornozelo. Essa limitação deve estar relacionada ao fato de que o programa de treinamento de força foi iniciado apenas na $6^{\text {a }}$ semana de PO (4a semana de fisioterapia) em função do risco de que a aplicação precoce de carga com faixa elástica ou peso corporal pudesse gerar para o tendão suturado. Assim, programas mais longos de fisioterapia, onde o treinamento muscular possa ser mantido com uma progressão temporal, ou programas com uma sobrecarga um pouco superior à utilizada no presente estudo, são talvez necessários para recuperar a força dos FP. 
CONCLUSÕES

O aumento do desequilíbrio muscular decorrente da lesão e imobilização após a cirurgia de reconstrução do TC refletiu-se na diminuição do torque dos FP.
A mobilização precoce do tornozelo no grupo ACE não acelerou o processo de recuperação, talvez devido à insuficiente sobrecarga mecânica sobre os FP aplicada durante o programa de fisioterapia precoce. Três meses de PO são insuficientes para o retorno aos mesmos níveis de produção de força dos músculos FP. Não parece existir necessidade de reabilitação dos FD do tornozelo, uma vez que eles não parecem ser afetados pela imobilização da articulação talocrural.

\section{REFERÊNCIAS}

1 Leppilahti J, Puranen J, Orava S. Incidence of Achilles tendon rupture. Acta Orthop Scand. 1996;67:277-9.

2 Maffulli N, Waterston SW, Squair J, Reaper J, Douglas AS. Changing incidence of Achilles tendon rupture in Scotland: a 15 years study. Clin J Sport Med. 1999;9(3):157-60.

3 Pajala A, Kangas J, Ohtonen P, Leppilahti J. Rerupture and deep infection following treatment of total Achilles tendon rupture. J Bone Joint Surg. 2002;84:2016-21.

4 Niixus SA, Nilsson B, Westlin N. The incidence of Achilles tendon rupture. Acta Orthop Scand. 1976;47(1):118-21.

5 Leppilahti J, Forsman K, Orawa S. Outcome and prognostic factors of Achilles rupture repair using a new scoring method. Clin Orthop Relat Res.1998;346:152-61.

6 Kannus P, Jozta L. Histopatological changes preceding spontaneous rupture of a tendon: a controlled study of 891 patients. J Bone Joint Surg. 1991;73:1507-25.

7 Stevens JE, Walter GA, Okereke E, Scarborough MT, Estherai JL, George SZ, et al. Muscle adaptations with immobilization and rehabilitation after ankle fracture. Med Sci Sports Exerc. 2004;36(10):1695-701.

8 Pathare NC, Stevens JE, Walter GA, Shah P, Jayamaran A, Tillman SM, et al. Deficit in human muscle strength with cast immobilization: contribution of inorganic phosphate. Eur J Appl Physiol. 2006;98(1):71-8.

9 Booth FW. Physiologic and biochemical effects of immobilization on muscle. Clin Orthop Relat Res. 1987;219:15-20.

10 Booth FW. Effect of limb immobilization on skeletal muscle. J Appl Physiol. 1982;52:1113-8.

11 Maffuli N, Tallon C, Wong J, Lim KP, Bleakney R. Early weightbearing and ankle mobilization after open repair of acute midsubstance tears of the Achilles tendon. Am J Sports Med. 2003;31(5):692-700.

12 Gomes CTS. Ruptura do tendão de Aquiles: tratamento cirúrgico, mobilização e cargas precoces. Rev Bras Ortop. 1998;33(12):951-8.

13 Mathias S, Kay K. Early full weightbearing and functional treatment after surgical repair of acute Achilles tendon rupture. Am J Sports Med. 1998;26(6):789-93.
14 Poulis S, Poulis I, Soames RW. Torque characteristics of the ankle plantarflexors and dorsiflexors during eccentric and concentric contraction in healthy young males. Isokinet Exerc Sci. 2000;8(4):195-202.

15 Fugl-Meyer AR. Maximum isokinetic ankle plantar and dorsal flexor torques in trained subjects. Eur J Appl Physiol. 1981;45:221-34.

16 So $\mathrm{CH}$, Siu TO, Chan KM, Chin MK. Isokinetic profile of dorsiflexors and plantarflexors of the ankle: a comparative study of élite vs untrained subjects. $\mathrm{Br} J$ Sports Med. 1994;28(1):25-30.

17 Krackow KA, Thomas SC, Jones LC. A new stitch for ligament-tendon fixation. J Bone Joint Surg Am. 1986;68:764-6.

18 Kerkhoffs GMMJ, Struijs PAA, Raaymakers ELBF, Marti RK. Functional treatment after surgical repair of acute Achilles tendon rupture: wrap vs walking cast. Arch Orthop Trauma Surg. 2002;122(2):102-5.

19 Vaz MA, Fração VB, Pressi MAS, Scheeren EM. Adaptação funcional do sistema musculoesquelético a demandas funcionais específicas: o caso dos movimentos repetitivos no trabalho. In: Merlo ARC, organizador. Saúde e trabalho no Rio Grande do Sul: realidade, pesquisa e intervenção. Porto Alegre: Ed. UFRGS; 2004. p.215-67.

20 Suchak AA, Spooner C, Reid DC, Jomha NM. Postoperative rehabilitation protocols for Achilles tendon ruptures: a meta-analysis. Clin Orthop Relat Res. 2006;445:216-21.

21 Wong J, Barrass V, Maffulli N. Quantitative review of operative and nonoperative management of Achilles tendon ruptures. Am J Sports Med. 2002;30(4):565-75.

22 Bhandari M, Guyatt GH, Siddiqui F, Morrow F, Busse J, Leighton RK, et al. Treatment of acute Achilles tendon ruptures: a systematic overview and metaanalysis. Clin Orthop Relat Res. 2002;400:190-200.

23 Khan RJK, Fick D, Brammar TJ, Crawford J, Parker MJ. Interventions for treating acute Achilles tendon ruptures. Cochrane Database Syst Rev. 2009;21:CD003674.

24 Speck M, Klaue K. Early full weightbearing and functional treatment after surgical repair of acute Achilles tendon rupture. Am J Sports Med. 1998;26:789-93. 